\title{
Scaling laws in velocity-selective coherent population trapping in the presence of polarization-gradient cooling
}

\author{
C. Menotti, G. Morigi, J. H. Müller, and E. Arimondo \\ Unità INFM, Dipartimento di Fisica, Università di Pisa, Piazza Torricelli 2, I-56126 Pisa, Italy
}

(Received 14 April 1997)

\begin{abstract}
One-dimensional laser cooling based on velocity-selective coherent population trapping (VSCPT) on a $2_{g} \rightarrow 1_{e}$ transition has been investigated numerically through the solution of the optical Bloch equations. As in the work of G. Morigi et al. [Phys. Rev. A 53, 2616 (1996)], it has been found that for a large set of atomic and laser parameters, the VSCPT cooling process may be described through scaling-law relations. The scaling laws are based on the relations between the loss rates at large atomic momentum and their dependence on the momentum around zero value. The role of the laser detuning on the VSCPT trapping efficiency has been examined and scaling laws including the detuning have been derived. [S1050-2947(97)01111-6]
\end{abstract}

PACS number(s): 32.80.Pj

Velocity-selective coherent population trapping (VSCPT) laser cooling is based on the preparation of atoms into a coherent superposition of ground states noncoupled to the applied laser fields. Efficient VSCPT cooling is realized if the coherent superposition is also a trap state being stable against kinetic energy evolution. In the $1_{g} \rightarrow 1_{e}$ transition of the experiment in helium $[1,2]$, the three-level $\Lambda$ scheme had the required stability and efficiency. An experimental investigation of rubidium atoms [3], also dealing with the $1_{g} \rightarrow 1_{e}$ transition, combined VSCPT with polarization-gradient cooling acting on another transition. VSCPT laser cooling on high $J$ transitions has been examined theoretically [4]. An important step towards the application of VSCPT on high $J$ transitions has been recently performed by some of us, with the derivation of scaling laws for the $1_{g} \rightarrow 1_{e}$ and $2_{g} \rightarrow 2_{e}$ atomic transitions $[5,6]$. The scaling laws link the VSCPT behavior for different atomic and angular momentum transitions. Thus the results obtained for one particular transition, for instance, the $1_{g} \rightarrow 1_{e}$ one in helium, allow us to predict the VSCPT results for other atoms and other angular momentum transitions. The idea of the scaling laws is based on an interpretation of VSCPT dynamics, introduced by Bardou et al. [7], as a Lévy-flight evolution in atomic momentum space. Within this point of view, three regions are identified in atomic momentum space: (i) the so-called Raman hole, that is, the momentum region that characterizes the filling of the trap state noncoupled to radiation, (ii) the momentum region outside the hole with a constant momentum diffusion rate, (iii) the high momentum region with a decreasing laser absorption rate due to Doppler effect. The scaling laws are based on the study of the noncoupled state absorption rate and the determination of the relation between the parameters characterizing the extension of the Raman hole and the momentum diffusion rate. Although the functional dependence of those quantities gets more complicated as the angular momentum increases, the regions of the Raman hole and of the momentum diffusion can still be identified.

In the present work VSCPT on the $2_{g} \rightarrow 1_{e}$ transition is examined and compared to the previously studied transitions. The $2_{g} \rightarrow 1_{e}$ transition has several original features. Using the standard $\sigma^{+}-\sigma^{-}$laser configuration the atomic evolu- tion is determined by two sets of momentum families, i.e., two sets of states closed for their time evolution under atomlaser interaction. One family is fully equivalent to that of the $\Lambda$ scheme in $1_{g} \rightarrow 1_{e}$ transition. For the other family, equivalent to that of the $2_{g} \rightarrow 2_{e}$ transition, a stable VSCPT superposition is realized when the kinetic energy difference between the states of the superposition is compensated by light shifts [4]. Thus, VSCPT in $2_{g} \rightarrow 1_{e}$ is characterized by a competition between two trap states. The present analysis demonstrates that for a laser resonant with the $2_{g} \rightarrow 1_{e}$ atomic transition VSCPT can be fitted by the previous scaling laws. Furthermore we have framed more precisely the scaling-law relations through the dependence of the groundstate loss rate on the atomic momentum, so that extension to any atomic transition is straightforward. A suitably detuned laser driving the $2_{g} \rightarrow 1_{e}$ or $2_{g} \rightarrow 2_{e}$ transition leads to polarization gradient cooling [8]. Thus, for this case VSCPT is assisted by cooling forces. In the present study we have also investigated the validity of the VSCPT scaling laws for the case of a detuned laser.

We consider atoms with mass $M$ and excited state spontaneous decay rate $\Gamma$, interacting with a laser field of frequency $\omega_{L}$ and wave number $k$, i.e., with recoil frequency $\omega_{R}=\hbar k^{2} / 2 M$. For $\sigma^{+}-\sigma^{-}$counterpropagating laser fields of amplitude $E$ interacting with the $2_{g} \rightarrow 1_{e}$ atomic transition, using the rotating-wave approximation (RWA) we write the atom-laser interaction as

$$
\begin{aligned}
V= & \frac{\hbar \Omega e^{-i \omega_{L} t}}{2} \\
& \times \sum_{p,(m=-2,2)}\left[\langle 1, m+1 ; 1,1 \mid 2, m\rangle\left|e_{p, m+1}\right\rangle\left\langle g_{p, m}\right|\right. \\
& \left.+\langle 1, m-1 ; 1,-1 \mid 2, m\rangle\left|e_{p, m-1}\right\rangle\left\langle g_{p, m}\right|\right]+ \text { H.c. }
\end{aligned}
$$

where $\left|a_{p, m}\right\rangle$, with $a=(g, e)$, describes a ground or excited state with magnetic quantum number $m$ and momentum $p+m \hbar k$. The Rabi frequency $\Omega=d E / \hbar$ is defined on the basis of the reduced dipole moment $d$ between upper and lower states [5]. We introduce the saturation parameter $s_{0}$ characterizing the atom-laser coupling 


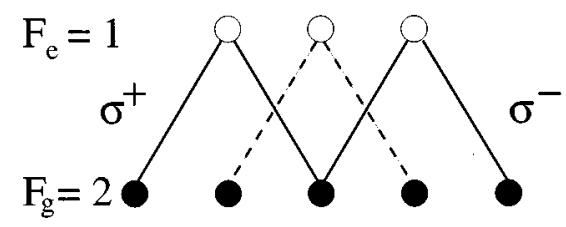

FIG. 1. Schematic representation of the $2_{g} \rightarrow 1_{e}$ transition excited by counterpropagating traveling-wave $\sigma^{+}$and $\sigma^{-}$light beams.

$$
s_{0}=\frac{2 \Omega^{2} / \Gamma^{2}}{1+4 \delta^{2} / \Gamma^{2}}
$$

with $\delta=\omega_{L}-\omega_{0}$ the detuning between the laser frequency and the atomic transition $\omega_{0}$. The $2_{g} \rightarrow 1_{e}$ coherently driven atomic transitions relevant for the VSCPT evolution are indicated in Fig. 1. The atomic states are classified into two independent families $\mathcal{F}_{\Lambda}(p)$ and $\mathcal{F}_{\mathrm{IW}}(p)$ composed of the following states, respectively:

$$
\begin{gathered}
\left|g_{p,-1}\right\rangle,\left|e_{p, 0}\right\rangle,\left|g_{p, 1}\right\rangle, \\
\left|g_{p,-2}\right\rangle,\left|e_{p,-1}\right\rangle,\left|g_{p, 0}\right\rangle,\left|e_{p, 1}\right\rangle,\left|g_{p, 2}\right\rangle .
\end{gathered}
$$

The noncoupled states associated to those families are

$$
\begin{gathered}
\left|\Phi^{\mathrm{NC}}(p)\right\rangle_{\Lambda}=\frac{1}{\sqrt{2}}\left[\left|g_{p,-1}\right\rangle-\left|g_{p, 1}\right\rangle\right], \\
\left|\Phi^{\mathrm{NC}}(p)\right\rangle_{\mathrm{IW}}=\frac{1}{2 \sqrt{2}}\left[\left|g_{p,-2}\right\rangle-\sqrt{6}\left|g_{p, 0}\right\rangle+\left|g_{p, 2}\right\rangle\right] .
\end{gathered}
$$

If the kinetic energy difference $4 \hbar \omega_{R}$ among the states composing the $\left|\Phi^{\mathrm{NC}}(p)\right\rangle_{\mathrm{IW}}$ superposition is compensated by a light shift produced by additional $\pi$-polarized light, both $\left|\Phi^{\mathrm{NC}}(p)\right\rangle_{\Lambda}$ and $\left|\Phi^{\mathrm{NC}}(p)\right\rangle_{\mathrm{IW}}$ are stable trap states [4]. In the following we will assume there is a compensation of the kinetic energy mismatch. In the limit of small Rabi frequency, i.e., $\Omega \ll|\delta-i \Gamma / 2|$ the atomic evolution may be expressed through the effective Hamiltonian based on the adiabatic elimination of the excited states. From the diagonalization of the effective Hamiltonian acting on the ground states of the $\mathcal{F}_{\Lambda}(p)$ and $\mathcal{F}_{\text {IW }}(p)$ families we derive the eigenvalues $E_{a}^{\mathrm{NC}}(p, \delta)$, with $a=\Lambda$ or IW, associated to the eigenstates of Eq. (5). These complex eigenvalues contain the energy shift $\delta_{a}^{\mathrm{NC}}(p, \delta)$, and the decay rate $\Gamma_{a}^{\mathrm{NC}}(p, \delta)$ :

$$
E_{a}^{\mathrm{NC}}(p, \delta)=\delta_{a}^{\mathrm{NC}}(p, \delta)-i \Gamma_{a}^{\mathrm{NC}}(p, \delta) .
$$

Results for the loss rates $\Gamma_{2 \rightarrow 1, \Lambda}^{\mathrm{NC}}$ and $\Gamma_{2 \rightarrow 1, \mathrm{IW}}^{\mathrm{NC}}$ at $\delta=0$ as a function of $p$ are shown in Fig. 2. Figure 2 shows also for comparison the loss rate $\Gamma_{1 \rightarrow 1}^{\mathrm{NC}}(p, \delta=0)$ for the $1_{g} \rightarrow 1_{e}$ transition, as derived in $[5,9]$. The loss rates have been expressed in units of $\Gamma^{\prime}=\Omega^{2} / 2 \Gamma$, the on-resonance optical pumping rate, while the Rabi frequency for each atomic transition is determined by the appropriate reduced dipole moment.

The scaling laws allow us to compare different atomic systems when the laser-atom interaction times are measured in units of the inverse of the $p \rightarrow \infty$ asymptotic value of the loss rate neglecting the Doppler shift. In effect the scaling

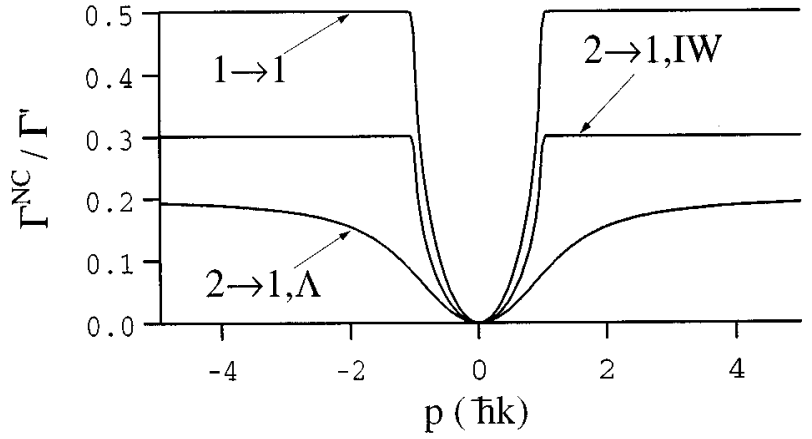

FIG. 2. Loss rates $\Gamma_{F_{g} \rightarrow F_{g}}^{\mathrm{NC}}(p, \delta=0) / \Gamma^{\prime}$ plotted vs $p / \hbar k$ for the $1_{g} \rightarrow 1_{e}$ transition and the kinetic energy compensated $2_{g} \rightarrow 1_{e}$ transition, at $\alpha_{1 \rightarrow 1}=1$ and $\alpha_{2 \rightarrow 1}^{\Lambda}=\alpha_{1 \rightarrow 1}^{\mathrm{IW}}=1$, respectively.

laws are valid when the initial momentum distribution is narrow, and for interaction times short enough to neglect atomic diffusion to momentum values where the Doppler shift is significant [5]. The asymptotic values of the loss rates are

$$
\begin{gathered}
\Gamma_{2 \rightarrow 1, \Lambda}^{\mathrm{NC}}(p \rightarrow \infty, \delta)=3 \Gamma s_{0} / 40 \\
\Gamma_{2 \rightarrow 1, \mathrm{IW}}^{\mathrm{NC}}(p \rightarrow \infty, \delta)=\Gamma s_{0} / 20
\end{gathered}
$$

while the asymptotic loss rates for the $1_{g} \rightarrow 1_{e}$ and $2_{g} \rightarrow 2_{e}$ transitions are

$$
\begin{gathered}
\Gamma_{1 \rightarrow 1}^{\mathrm{NC}}(p \rightarrow \infty, \delta)=\Gamma s_{0} / 8, \\
\Gamma_{2 \rightarrow 2}^{\mathrm{NC}}(p \rightarrow \infty, \delta)=\Gamma s_{0} / 12 .
\end{gathered}
$$

The plot of the loss rates around the $p=0$ value in Fig. 2 shows the characteristic minimum denoted as the Raman hole $[5,10]$. The VSCPT optical pumping rate into the $\left|\Phi^{\mathrm{NC}}(p=0)\right\rangle$ trap states is determined by the extension of the Raman hole in the momentum space. In order to compare the Raman holes for different transitions, we write for $p \approx 0$

$$
\Gamma_{F_{g} \rightarrow F_{e}}^{\mathrm{NC}}(p \approx 0) \approx \frac{\Gamma_{F_{g} \rightarrow F_{e}}^{\mathrm{NC}}(p \rightarrow \infty, \delta)}{2}\left[\frac{p}{\alpha_{F_{g} \rightarrow F_{e}}(\delta) \hbar k}\right]^{2} .
$$

This equation defines $\alpha_{F_{g} \rightarrow F_{e}}(\delta)$ as the extension of the Raman hole. An analytical calculation of the eigenvalues shows that the loss rate $\Gamma_{F_{g} \rightarrow F_{e}}^{\mathrm{NC}}(p \approx 0)$ does not depend on the laser detuning $\delta$. The definition of the Raman hole width by Eq. (9) in terms of the asymptotic loss rate, which depends on the detuning, introduces a dependence of $\alpha_{F_{g} \rightarrow F_{e}}$ on $\delta$. The following relations apply:

$$
\begin{gathered}
\alpha_{1 \rightarrow 1}(\delta)=\frac{\Gamma}{\omega_{\mathrm{R}}} \frac{s_{0} \sqrt{1+4 \delta^{2} / \Gamma^{2}}}{16}, \\
\alpha_{2 \rightarrow 1}^{\Lambda}(\delta)=\frac{3}{5} \frac{\Gamma}{\omega_{\mathrm{R}}} \frac{s_{0} \sqrt{1+4 \delta^{2} / \Gamma^{2}}}{16}, \\
\alpha_{2 \rightarrow 1}^{\mathrm{IW}}(\delta)=\frac{\sqrt{6}}{5} \frac{\Gamma}{\omega_{\mathrm{R}}} \frac{s_{0} \sqrt{1+4 \delta^{2} / \Gamma^{2}}}{16},
\end{gathered}
$$



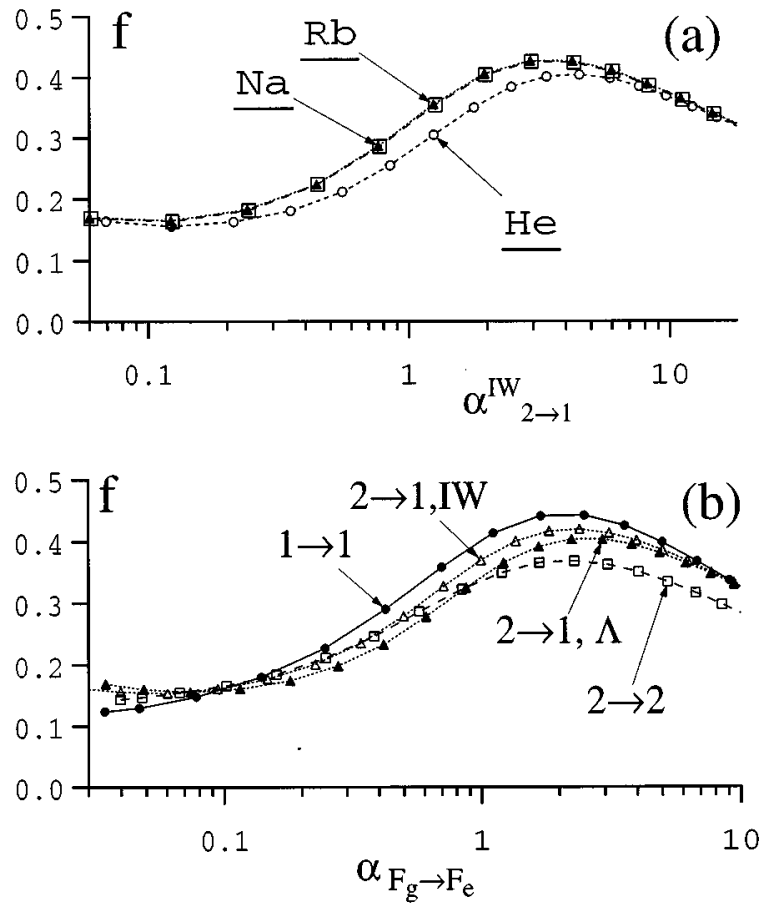

FIG. 3. In (a) trapped fraction $f$ for the $2_{g} \rightarrow 1_{e}$ transition and helium, sodium, and rubidium parameters vs $\alpha_{2 \rightarrow 1}^{\mathrm{IW}}$. In (b) trapped fraction $f$ of helium vs $\alpha_{F_{g} \rightarrow F_{e}}$ for different atomic transitions. Interaction times $\Theta \Gamma_{F_{g} \rightarrow F_{e}}^{\mathrm{NC}}(p \rightarrow \infty)=132 \alpha$ and initial distribution width $\delta p_{0}=1 \hbar k$ in all cases.

$$
\alpha_{2 \rightarrow 2}(\delta)=\sqrt{\frac{2}{27}} \frac{\Gamma}{\omega_{\mathrm{R}}} \frac{s_{0} \sqrt{1+4 \delta^{2} / \Gamma^{2}}}{16} .
$$

For the different atoms and/or different optical transitions VSCPT occurs with equal efficiency (i) for equal Raman hole extension, i.e., if the Rabi frequencies are chosen in order to match the $\alpha$ values of the corresponding transitions, and (ii) for equal effective interaction times, i.e., if the times measured in units of the inverse of the limiting loss rates are equal. In [5] the comparison between the $1_{g} \rightarrow 1_{e}$ and $2_{g} \rightarrow 2_{e}$ transitions was based on a heuristic scaling of $\alpha_{2 \rightarrow 2}$ by a factor of four, corresponding to the increase in kinetic energy of the $2_{g} \rightarrow 2_{e}$ trap state. The present approach provides a more solid relation for the scaling factors between those atomic transitions, as well for all others.

Figures 3 and 4 show the VSCPT efficiency for different atoms and optical transitions versus the $\alpha_{F_{g} \rightarrow F_{e}}$ parameters. The VSCPT efficiency is expressed by the fraction $f$ of atoms trapped in the states of the $\mathcal{F}_{\Lambda}(p)$ or $\mathcal{F}_{\text {IW }}(p)$ families with $|p| \leqslant \hbar k / 10$. The trapped fractions, obtained through the solution of optical Bloch equations, are smaller than those obtained through the quantum Monte Carlo analysis [5]. The interaction times $\Theta$ of Figs. 3 and 4 satisfy the following relation: $\Theta \Gamma_{F_{g} \rightarrow F_{e}}^{\mathrm{NC}}(p \rightarrow \infty)=132 \alpha$, leading to a constant value of the interaction time when expressed in terms of $1 / \omega_{\mathrm{R}}$. Figure 3(a) shows the $2_{g} \rightarrow 1_{e}$ trapped fraction $f$ for helium, sodium, and rubidium parameters versus $\alpha_{2 \rightarrow 1}^{\mathrm{IW}}$ in the case of $\delta=0$ and an initial momentum distribution width of $1 \hbar k$. It may be noticed that the application of the scaling law leads to a good agreement for the VSCPT results of different
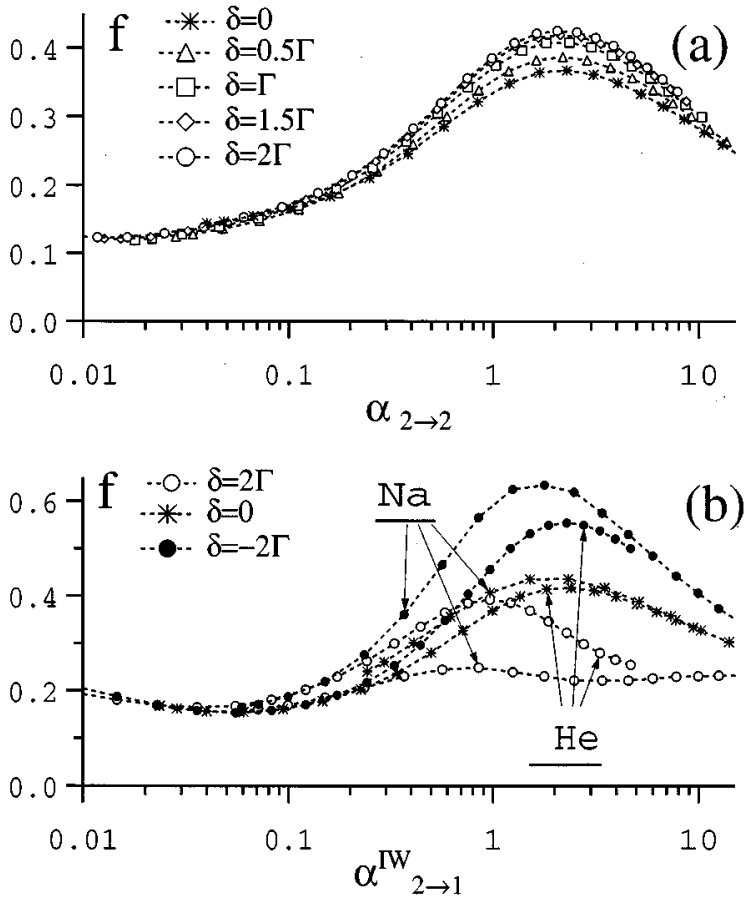

FIG. 4. In (a) trapped fraction $f$ for $2_{g} \rightarrow 2_{e}$ transition and helium parameters vs the $\alpha_{2 \rightarrow 2}$ at different laser detunings. Only blue detunings have been shown, because within the accuracy of our data the trapped fraction depends on the absolute value of $\delta$. In (b) trapped fraction $f$ of helium and sodium atoms vs $\alpha_{2 \rightarrow 1}^{M}$. Interaction times $\Theta \Gamma_{F_{g} \rightarrow F_{e}}^{\mathrm{NC}}(p \rightarrow \infty)=132 \alpha$ and initial distribution width $\delta p_{0}=1 \hbar k$ in all cases.

atoms. The smaller value of $f$ for helium parameters, consistent with the $1_{g} \rightarrow 1_{e}$ results of [5], is originated by the presence of a large Doppler shift for this light atom. Figure 3(b) shows results for the trapped fraction of different transitions versus the $\alpha_{F_{g} \rightarrow F_{e}}$ parameter, again at laser detuning $\delta=0$. By applying the scaling laws, those data, obtained for helium, may be applied to other atoms. For the $2_{g} \rightarrow 1_{e}$ transition two different scalings are obtained using the $\alpha_{2 \rightarrow 1}^{\Lambda}$ or $\alpha_{2 \rightarrow 1}^{\text {IW }}$ values.

The quantitative basis for the scaling laws defined by Eqs. (7), (8), and (10) allows us to compare VSCPT in the presence of laser detuning. Figure 4 shows results for the trapped fraction $f$ versus the $\alpha$ parameter at different values of the detuning and for different atomic transitions. Let us notice that application of the scaling law for different detunings requires comparing the trapped fractions at different interaction times. Scaling the interaction time $\Theta(\delta)$ with the limiting loss rates of Eqs. (7) or (8), we obtain $\Theta(\delta)=\Theta(0) \sqrt{1+4 \delta^{2} / \Gamma^{2}}$. In Fig. 4(a) the trapped fraction of the $2_{g} \rightarrow 2_{e}$ transition is shown for the case of helium parameters at different laser detunings on the blue side because, when a narrow initial momentum distribution is used, the VSCPT efficiency depends only very weakly on the sign of the detuning. For the $2_{g} \rightarrow 2_{e}$ transition the scaled VSCPT efficiency presents only a weak dependence on the laser detuning. A similar result applies also for the $1_{g} \rightarrow 1_{e}$ transition. Figure 4(b) shows results for helium and sodium on the $2_{g} \rightarrow 1_{e}$ transition at different red and blue laser detunings, taking into account the population in both trap states. For this 
transition even applying the scaling for the interaction time and the Raman hole extension, the VSCPT efficiency presents a strong dependence on the detuning. Moreover for detunings $\delta \neq 0$ the independence of the atomic parameters, derived in Fig. 3 in the case of zero detuning, is no longer valid.

The interpretation of the results of Fig. 4 requires investigating the role of the polarization-gradient cooling and of the Lévy-flight evolution. Polarization-gradient cooling for the $2_{g} \rightarrow 2_{e}$ transition is not very efficient [8]. The results of Fig. 4(a) with the trapped fraction independent of the detuning, evidence that, at least for a narrow initial atomic momentum distribution, the polarization gradient cooling has a small influence on the VSCPT efficiency of the $2_{g} \rightarrow 2_{e}$ transition. For the $2_{g} \rightarrow 1_{e}$ transition, the analysis of Ref. [8] suggested an efficient process of polarization-gradient cooling. The results for sodium and the helium atom of Fig. 4(b) evidence that the dependence of the polarization-gradient cooling on the detuning derived in Ref. [8] does not apply to VSCPT in the case where the ground-state kinetic energy mismatch is compensated by the light shift. An additional result of Fig. 4(b) is the large difference in the sodium and helium VSCPT efficiencies, so that the scaling laws are not satisfied for the case of $2_{g} \rightarrow 1_{e}$ VSCPT with a detuned laser. This anomalous behavior is originated by the presence of two trap states, one within the $\Lambda$ family with zero cooling force, the other one within the IW family with cooling force different from zero. Through the spontaneous emissions, at- oms jump between the two families, and the competition between the Lévy-flight evolution of the two trap states produces the dependence on atomic species and laser detuning of Fig. 4(b).

Scaling laws have been derived for the dependence of the fraction of trapped atoms on the interaction time and the Rabi frequency for different atomic transitions. We have extended our previous study to the case of a $F \rightarrow F-1$ transition. Furthermore we have derived a quantitative basis for the scaling between different transitions using the Raman hole extension and the dependence of the loss rate on the atomic momentum. In the absence of polarization-gradient force the scaling laws between different atomic transitions is very good. The presence of a strong polarization gradient force weakens the validity of the scaling relations, in particular when several trap states are present, because of the competition between trap states with comparable occupations. In any case the scaling laws provide good estimates of the laser parameters to be used for realizing an efficient VSCPT trapping.

This study was supported by the European Community, through TMR Grant No. ERB4061PL95-0044, and the Consiglio Nazionale delle Ricerche of Italy. J.H.M. acknowledges the European Community for financial support. Two authors (C.M. and E.A.) wish to thank C. Cohen-Tannoudji for a useful discussion.
[1] A. Aspect, E. Arimondo, R. Kaiser, N. Vansteenkiste, and C. Cohen-Tannoudji, Phys. Rev. Lett. 61, 826 (1988); J. Opt. Soc. Am. B 6, 2112 (1989).

[2] J. Lawall, F. Bardou, B. Saubamea, K. Shimizu, M. Leduc, A. Aspect, and C. Cohen-Tannoudji, Phys. Rev. Lett. 73, 1915 (1994); J. Lawall, S. Kulin, B. Saubamea, N. Bigelow, M. Leduc, and C. Cohen-Tannoudji, ibid. 75, 4194 (1995).

[3] T. Esslinger, F. Sander, M. Weidenmüller, A. Hemmerich, and T. W. Hänsch, Phys. Rev. Lett. 76, 2432 (1996).

[4] M. A. Ol'Shanii, J. Phys. B 24, 2583 (1991); Opt. Spectrosc. 76, 174 (1994); C. J. Foot, H. Wu, E. Arimondo, and G. Morigi, J. Phys. (France) II 4, 1913 (1994).

[5] G. Morigi, B. Zambon, N. Leinfellner, and E. Arimondo, Phys.
Rev. A 53, 2616 (1996).

[6] VSCPT scaling laws can be derived also from the analysis presented in S. Schauffler and V. P. Yakovlev, Laser Phys. 6, 414 (1996).

[7] F. Bardou, J. P. Bouchaud, O. Émile, A. Aspect, and C. Cohen-Tannoudji, Phys. Rev. Lett. 72, 203 (1994).

[8] A. M. Steane, G. Hillebrand, and C. J. Foot, J. Phys. B 25, 4721 (1992); J. Werner, H. Wallis, G. Hillebrand, and A. Steane, ibid. 26, 3063 (1993).

[9] A factor 0.5 missing in the plot of $\Gamma_{1 \rightarrow 1}^{\mathrm{NC}}(p, \delta=0)$ in Fig. 2(a) of [5] has been introduced here in Fig. 2.

[10] P. Marte, R. Dum, R. Tä̈eb, P. Zoller, M. S. Shahriar, and M. Prentiss, Phys. Rev. A 49, 4826 (1994). 